\title{
KNOTS, GROUPS, AND SPINNING
}

\author{
by CHERRY KEARTON
}

(Received 12 October, 1989)

In this note we observe how results of Gordon, Luecke, Feustel, and Whitten establish the precise extent to which knowledge of the knot group determines the knot, in both the oriented and the unoriented case. This enables us to say, in terms of the knot group, exactly when two spun knots are the same.

We work throughout in the smooth or piecewise-linear category. Let $k$ be an oriented knot $\left(S^{3}, S^{1}\right)$, and let $K$ be the closed complement in $S^{3}$ of a tubular neighbourhood $S^{1} \times B^{2}$ of $S^{1}$. The orientations of $S^{1}$ and $S^{3}$ give rise to a natural choice of meridian $m$, being represented by point $\times S^{1} \subset S^{1} \times S^{1}=\partial\left(S^{1} \times B^{2}\right)$; so that $S^{1}$ and $m$ have linking number +1 . We shall think of $m$ as a conjugacy class in $\pi_{1}(K)$, and denote by $\pi_{1}(k)$ the pair $\left(\pi_{1}(K), m\right)$. A useful reference for this and what follows is [2].

We denote by $k^{-1}$ the knot obtained by reversing the orientation of $S^{1}$, by $k^{*}$ the mirror image of $k$, and by $-k$ the result of reversing the orientations of both $S^{1}$ and $S^{3}$. If $l$ denotes a longitude of $k$, represented by $S^{1} \times$ point $\subset S^{1} \times S^{1}=\partial\left(S^{1} \times B^{2}\right)$, having linking number zero with $S^{1}$ and homologous to $k$ in $S^{1} \times B^{2}$, then we note the following relationships between the longitudes and meridians of these knots.

$$
\begin{array}{cl}
k & (l, m) \\
k^{-1} & \left(l^{-1}, m^{-1}\right) \\
k^{*} & \left(l, m^{-1}\right) \\
-k & \left(l^{-1}, m\right) .
\end{array}
$$

Two unoriented knots $k$ and $h$ are equivalent if there is a homeomorphism taking one pair onto the other; in the case of oriented knots we require that the homeomorphism should also preserve orientations. Such a homeomorphism, of course, induces an isomorphism of pairs $\pi_{1}(k) \cong \pi_{1}(h)$.

If we think of the pair $(l, m)$ as determined up to conjugacy in $\pi_{1}(K)$ then the pair $\left(\pi_{1}(K),(l, m)\right)$ determines the oriented knot $k$, up to equivalence, by a result of Waldhausen (see [2, p. 39]).

The following result is Theorem 1 of [3] in the composite case, and follows at once from Corollary 2 of [7] and Theorem 1 of [5] in the prime case.

THEOREM 1. Let $k$ and $h$ be unoriented knots with $\pi_{1}(K) \cong \pi_{1}(H)$. Then $k$ and $h$ factor into primes as $k=k_{1}+\ldots+k_{n}, h=h_{1}+\ldots+h_{n}$, with $k_{i}=h_{i}$ for each $i$.

THEOREM 2. Let $k$ and $h$ be oriented knots with $\pi_{1}(k) \cong \pi_{1}(h)$. Then $k$ and $h$ factor into primes as $k=k_{1}+\ldots+k_{n}, h=h_{1}+\ldots+h_{n}$, with $k_{i}= \pm h_{i}$ for each $i$.

Proof. In the case $n=1$, we see by Corollary 2 of [7] and Theorem 1 of [5] that $k$ and $h$ are equivalent as unoriented knots. Thus $k=h, h^{-1}, h^{*}$, or $-h$. But the isomorphism $\pi_{1}(k) \cong \pi_{1}(h)$ preserves meridians, and so we must have $k= \pm h$ (compare Proposition 3.19 of [2]).

In the case $n>1$, we appeal to Proposition 15.39 of [2] to see that after factorizing $k$ and $h$ we may assume that $\pi_{1}\left(k_{i}\right) \cong \pi_{1}\left(h_{i}\right)$, and hence $k_{i}= \pm h_{i}$ for each $i$.

Now we recall the operation of $p$-spinning, as described in [4], which yields a

Glasgow Math. J. 33 (1991) 99-100. 
$(p+1)$-knot $\sigma_{p}(k)$. We let $\left(B^{3}, B^{1}\right)$ be the closed complement of a regular neighbourhood of a point on $S^{1}$ : this is a knotted arc in $B^{3}$ which contains all the knottedness of $k$. Define $\sigma_{p}(k)$ to be the pair $\partial\left[\left(B^{3}, B^{1}\right) \times B^{p+1}\right]=\left(S^{p+3}, S^{p+1}\right)$. Choosing a fixed orientation for $B^{p+1}$, we can make $\sigma_{p}(k)$ oriented when $k$ is oriented.

THEOREM 3. (i) If $k$ and $h$ are oriented knots, then $\sigma_{p}(k)=\sigma_{p}(h)$ if and only if $\pi_{1}(k) \cong \pi_{1}(h)$, where $p \geqslant 1$.

(ii) If $k$ and $h$ are unoriented knots, then $\sigma_{p}(k)=\sigma_{p}(h)$ if and only if $\pi_{1}(K) \cong \pi_{1}(H)$, whe: $p \geqslant 1$.

Proof. (i) Since $p$-spinning preserves both knot group and meridian, the "only if" part is trivial. So assume that $\pi_{1}(k) \cong \pi_{1}(h)$. By Theorem $2, k$ and $h$ factor into primes with $k_{i}= \pm h_{i}$ for each $i$ and, by [4], we have

$$
\sigma_{p}(k)=\sigma_{p}\left(k_{1}+\ldots+k_{n}\right)=\sigma_{p}\left(k_{1}\right)+\ldots+\sigma_{p}\left(k_{n}\right)=\sigma_{p}\left(h_{1}\right)+\ldots+\sigma_{p}\left(h_{n}\right)=\sigma_{p}(h) .
$$

(ii) Again the "only if" part is trivial; so assume that $\pi_{1}(K) \cong \pi_{1}(H)$. Then $k$ and $h$ factorize as in Theorem 1 . If $n=1$ then $k$ and $h$ are equivalent and so $\sigma_{p}(k)=\sigma_{p}(h)$. If $n>1$, orient $k$ and $h$, then, by Proposition 15.39 of [2], the induced isomorphisms of $\pi_{1}\left(K_{i}\right)$ and $\pi_{1}\left(H_{i}\right)$ either consistently preserve or consistently reverse the meridians. In the latter case, reorient $h$ to $h^{-1}$ so that the meridians are preserved by the isomorphisms. Then $\pi_{1}(k) \cong \pi_{1}(h)$ and so $\sigma_{p}(k)=\sigma_{p}(h)$ by part (i).

\section{Corollary 1. The operation of p-spinning is finite-to-one.}

Proof. By [3, Corollary 4], [2, Proposition 15.40], the corollary is true for composite knots. It is also true for prime knots by [7, Corollary 2] and [5, Theorem 1].

Corollary 2. For $p, q \geqslant 1$,

$$
\sigma_{p}(k)=\sigma_{p}(h) \Leftrightarrow \sigma_{q}(k)=\sigma_{q}(h),
$$

in both the oriented and unoriented case.

The reader may care to compare these results with those of [1] and [6], where the spinning of certain high-dimensional knots is considered.

Finally, it is a pleasure to acknowledge the help and advice of J. A. Hillman, who was visiting Durham as an SERC Visiting Fellow when this paper was conceived.

\section{REFERENCES}

1. E. Bayer-Fluckiger, C. Kearton and S. M. J. Wilson, Finiteness theorems for conjugacy classes and branched covers of knots, Math. Z. 201 (1989) 485-493.

2. G. Burde and H. Zieschang, Knots (Walter de Gruyter, 1985).

3. C. D. Feustel and W. Whitten, Groups and complements of knots, Canad. J. Math. 30 (1978), 1284-1295.

4. C. McA. Gordon, A note on spun knots, Proc. Amer. Math. Soc. 58 (1976), 361-362.

5. C. McA. Gordon and J. Luecke, Knots are determined by their complements, preprint 1988, University of Texas at Austin.

6. C. Kearton, Simple spun knots, Topology 23 (1984), 91-95.

7. W. Whitten, Knot complements and groups, Topology 26 (1987), 41-44.

Department of Mathematical Sciences

DURHAM UNIVERSITY

Durham DH1 3LE

ENGLAND 